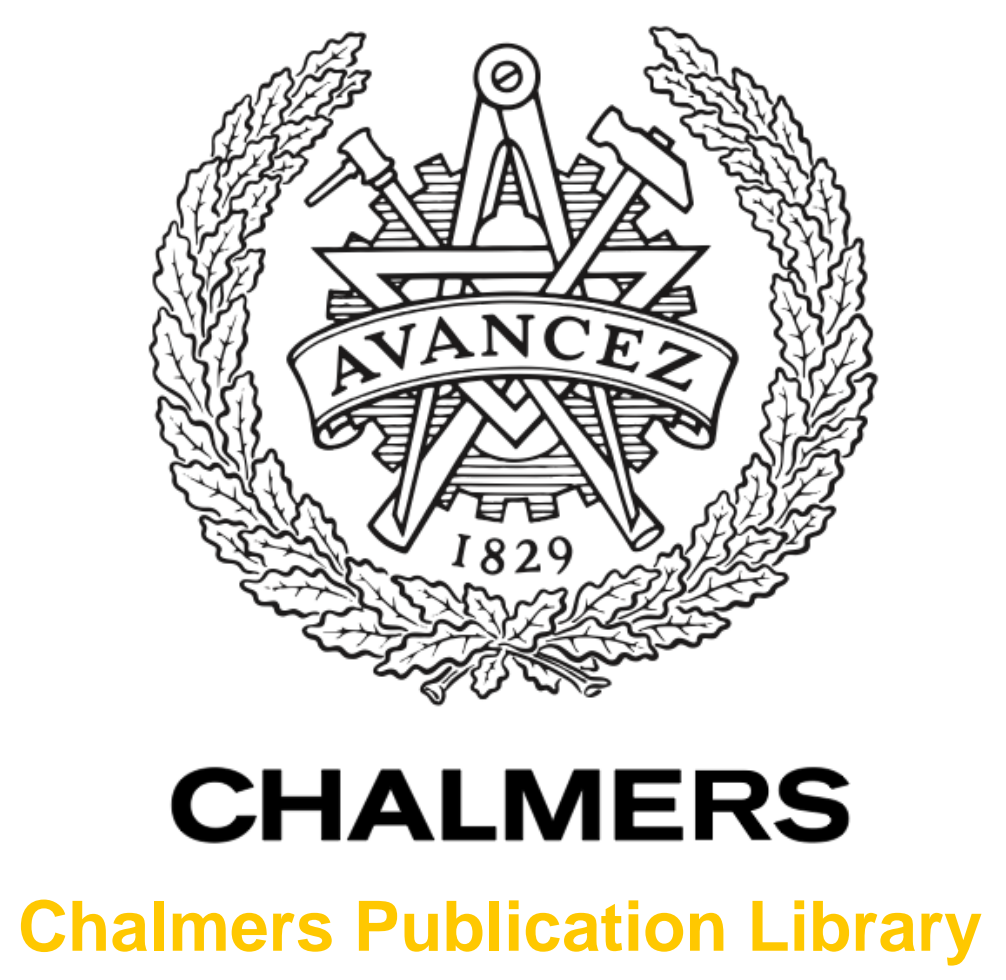

\title{
On the separation of timescales in radio-based positioning
}

This document has been downloaded from Chalmers Publication Library (CPL). It is the author's version of a work that was accepted for publication in:

\section{Localization and GNSS (ICL-GNSS), 2015 International Conference on}

Citation for the published paper:

Fröhle, M. ; Wymeersch, H. (2015) "On the separation of timescales in radio-based positioning". Localization and GNSS (ICL-GNSS), 2015 International Conference on pp. 16.

Downloaded from: http://publications.lib.chalmers.se/publication/225926

Notice: Changes introduced as a result of publishing processes such as copy-editing and formatting may not be reflected in this document. For a definitive version of this work, please refer to the published source. Please note that access to the published version might require a subscription. 


\title{
On the Separation of Timescales in Radio-based Positioning
}

\author{
Markus Fröhle and Henk Wymeersch \\ Department of Signals and Systems, Chalmers University of Technology, Gothenburg, Sweden \\ email: \{frohle,henkw\}@chalmers.se
}

\begin{abstract}
Radio-based positioning methods are generally based on a separation of timescales, where each position update assumes the availability of synchronous measurements. This approach is convenient in the design of positioning algorithms, but fails to account for outdated information. We describe three distinct ways how the separation of timescales can adversely impact the design and execution of positioning methods, and quantify the extent of these impacts analytically.
\end{abstract}

\section{INTRODUCTION}

The ability to position a mobile device (e.g., a mobile phone) through radio signals has numerous applications, not only for personal navigation, but also for location-based services, search-and-rescue operations, crowd sourcing, and location-aided communication. Positioning algorithms generally comprise three components: (i) a measurement stage in which relative position measurements are collected with respect to reference points or other mobile devices, (ii) a position update stage in which a position estimate is computed based on the measurements; and (iii) a prediction stage in which a location is estimated in the absence of measurements. The prediction stage is usually employed to track the device while it is moving and is an integral part of model based techniques such as, e.g., the Kalman filter.

A large part of the positioning literature has focused on static networks, and has developed performance bounds and sophisticated algorithms that operate close to those bounds. However, by ignoring mobility, there is no real notion of time, so that bounds and algorithms can perform arbitrarily well by simply collecting more measurements and running more complex algorithms. The aspect of time in positioning was studied in [1] in terms of the time needed to collect measurements. It was found that approaches which are most attractive when ignoring time become most cumbersome when time is accounted for. In the context of mobile networks, techniques based on the Kalman filter and its generalizations generally freeze time during the measurement and position update stages, thus suffering from the same problems as static networks. When measurements arrive out of order, the Kalman filter must be rolled back in order to deal with out-of-sequence measurements [2]. In [3], the effect of movement and outof-date measurements on the localization accuracy of mobile agents was investigated through an additional error term in the measurement model. It was shown that delays adversely affect the accuracy.

978-1-4799-9858-6/15/\$31.00 (C)2015 IEEE
In this paper, we study the impact of the assumption of separation of timescales from three different perspectives:

1) The impact on the Cramér-Rao bound, where we show that the separation of timescales leads to overly optimistic fundamental performance bounds;

2) The impact of out of date measurements on tracking, where we show that the separation of timescales leads to loss of integrity for mobile devices; and

3) The impact on message scheduling in cooperative positioning, where the separation of timescales leads to overly confident a posteriori distribution approximations.

Notation: Vectors are denoted in boldface and lower case (e.g., $\mathbf{x}$ ) and matrices in boldface upper case (e.g., A), $\mathbf{I}_{M}$ is a $M \times M$ identity matrix, $\mathbf{1}_{L}$ denotes a column vector of $L$ ones, $\operatorname{tr}\{\cdot\}$ denotes the trace of a matrix, $\otimes$ is the Kronecker product, $\Delta_{\mathbf{b}}^{\mathbf{a}} f(\mathbf{a}, \mathbf{b})=\nabla_{\mathbf{b}} \nabla_{\mathbf{a}}^{\mathrm{T}} f(\mathbf{a}, \mathbf{b})$, the $\ell_{2}$ norm is denoted $\|\cdot\|$.

\section{The Positioning Problem}

\section{A. General Version}

We state a version of the positioning problem that encompasses many standard models. We consider a network with $N+M$ nodes, comprising $N \geq 1$ mobile agents and $M \geq 3$ static anchors. The state of agent $n$ at time $t$ is denoted by $\mathbf{x}_{n}(t)$ and is governed by the process model of the form

$$
\dot{\mathbf{x}}_{n}(t)=\mathbf{f}_{n}\left(\mathbf{x}_{n}(t), \mathbf{w}_{n}(t), \mathbf{u}_{n}(t)\right),
$$

in which $\mathbf{f}_{n}(\cdot)$ is an arbitrary (possibly non-linear) function, which can include an external control input $\mathbf{u}_{n}(t)$ and can thus be time-dependent, and $\mathbf{w}_{n}(t)$ is process noise, assumed to be white in time and across nodes. Each agent $n$ can obtain measurements $\mathbf{z}_{n, k}(t)$ with respect to another node $k$. Measurements depend on the relative position between the nodes involved, e.g., distance or orientation, and can be expressed as

$$
\mathbf{z}_{n, k}(t)=\mathbf{h}_{n, k}\left(\mathbf{x}_{n}(t), \mathbf{x}_{k}(t), \mathbf{n}_{n, k}(t)\right),
$$

in which $\mathbf{h}_{n, k}(\cdot)$ is an arbitrary (possibly non-linear) function and $\mathbf{n}_{n, k}(t)$ is measurement noise. When agents collect measurements with respect to other agents, we say that the network is cooperative.

Given an a priori distribution at a starting time $t_{0}$, say $p\left(\mathbf{x}_{n}\left(t_{0}\right)\right)$, and the statistical models $p\left(\mathbf{x}_{n}(t) \mid \mathbf{x}_{n}\left(t^{\prime}\right)\right)$ for $t>t^{\prime}$ as well as the likelihood $p\left(\mathbf{z}_{n, k}(t) \mid \mathbf{x}_{n}(t), \mathbf{x}_{k}(t)\right)$, our goal is 
to determine $p\left(\mathbf{x}_{n}(t) \mid \mathbf{Z}_{\leq t}\right)$ in which $\mathbf{Z}_{\leq t}$ represents all the measurements up to and including time $t$.

\section{B. Simplified Version}

In order to gain insights, we will also consider a simplified scenario in which the state is the two-dimensional position $\mathbf{x}_{n}(t) \in \mathbb{R}^{2}$ and the process model is a constant velocity model, whereby

$$
\dot{\mathbf{x}}_{n}(t)=\mathbf{v}+\dot{\mathbf{w}}_{n}(t),
$$

in which $\mathbf{v}$ is a fixed velocity vector and $\dot{\mathbf{w}}_{n}(t) \sim$ $\mathcal{N}\left(\mathbf{0}, \sigma_{\text {mob }}^{2} \mathbf{I}\right)$ is velocity noise. It then follows that

$$
\mathbf{x}_{n}(t)=\mathbf{x}_{n}\left(t^{\prime}\right)+\left(t-t^{\prime}\right) \mathbf{v}+\mathbf{w}_{n}(t),
$$

where

$$
\begin{aligned}
\mathbf{w}_{n}(t) & =\int_{t^{\prime}}^{t} \dot{\mathbf{w}}_{n}(u) \mathrm{d} u \\
& \sim \mathcal{N}\left(\mathbf{0},\left(t-t^{\prime}\right) \sigma_{\mathrm{mob}}^{2} \mathbf{I}\right) .
\end{aligned}
$$

As a simplified measurement model, we consider

$$
\mathbf{z}_{n, k}(t)=\mathbf{x}_{n}(t)-\mathbf{x}_{k}(t)+\mathbf{n}_{n, k}(t),
$$

where $\mathbf{n}_{n, k}(t) \sim \mathcal{N}\left(\mathbf{0}, \sigma_{\text {meas }}^{2} \mathbf{I}\right)$. Finally, we will consider only discrete time instants with a resolution of $T_{s}$, so that measurements and predictions are only performed at times $t=t_{0}+k T_{s}, k \in \mathbb{N}_{>0}$.

\section{Sequential Positioning: Algorithm and Bound}

In this section we describe the optimal approach to determine $p\left(\mathbf{x}_{n}(t) \mid \mathbf{Z}_{\leq t}\right)$. We also review a fundamental performance bound.

\section{A. Algorithm: Bayesian Filtering}

We introduce $\mathbf{x}_{n}^{k}=\mathbf{x}_{n}\left(t_{0}+k T_{s}\right), \mathbf{X}^{k}=\left[\mathbf{x}_{1}^{k^{\mathrm{T}}}, \ldots, \mathbf{x}_{N}^{k^{\mathrm{T}}}\right]^{\mathrm{T}}$, and $\mathbf{X}^{0: k}=\left[\mathbf{X}^{0^{\mathrm{T}}}, \mathbf{X}^{1^{\mathrm{T}}}, \ldots, \mathbf{X}^{k^{\mathrm{T}}}\right]^{\mathrm{T}}$. Similarly, we group the measurements at time $k$ into $\mathbf{Z}^{k}$ and introduce $\mathbf{Z}^{1: k}=$ $\left[\mathbf{Z}^{1^{\mathrm{T}}}, \ldots, \mathbf{Z}^{k^{\mathrm{T}}}\right]^{\mathrm{T}}$. We tacitly assume that when no measurements are available at epoch $k$, then $\mathbf{Z}^{k}$ is empty. We can express the joint distribution of $\mathbf{X}^{0: k}$ and $\mathbf{Z}^{1: k}$ as

$$
\begin{aligned}
& p\left(\mathbf{X}^{0: k}, \mathbf{Z}^{1: k}\right) \\
& =p\left(\mathbf{X}^{0: k}\right) p\left(\mathbf{Z}^{1: k} \mid \mathbf{X}^{0: k}\right) \\
& =p\left(\mathbf{X}^{0}\right) \prod_{j=1}^{k} p\left(\mathbf{X}^{j} \mid \mathbf{X}^{j-1}\right) p\left(\mathbf{Z}^{j} \mid \mathbf{X}^{j}\right) \\
& =\prod_{n=1}^{N} p\left(\mathbf{x}_{n}^{0}\right) \prod_{j=1}^{k} p\left(\mathbf{x}_{n}^{j} \mid \mathbf{x}_{n}^{j-1}\right) \prod_{m \in \mathcal{R}_{n}^{j}} p\left(\mathbf{z}_{n, m}^{j} \mid \mathbf{x}_{n}^{j}, \mathbf{x}_{m}^{j}\right),
\end{aligned}
$$

where $\mathcal{R}_{n}^{j}$ is the set of measurement partners (agents or anchors) of agent $n$ at time $j$. The posterior distribution $p\left(\mathbf{X}^{k} \mid \mathbf{Z}^{1: k}\right)$ can be expressed as [4]

$$
\begin{aligned}
& p\left(\mathbf{X}^{k} \mid \mathbf{Z}^{1: k}\right) \\
& \propto p\left(\mathbf{Z}^{k} \mid \mathbf{X}^{k}\right) \int p\left(\mathbf{X}^{k-1} \mid \mathbf{Z}^{1: k-1}\right) p\left(\mathbf{X}^{k} \mid \mathbf{X}^{k-1}\right) \mathrm{d} \mathbf{X}^{k-1} \\
& =p\left(\mathbf{Z}^{k} \mid \mathbf{X}^{k}\right) p\left(\mathbf{X}^{k} \mid \mathbf{Z}^{1: k-1}\right)
\end{aligned}
$$

enabling a recursive computation with a prediction step to determine $p\left(\mathbf{X}^{k} \mid \mathbf{Z}^{1: k-1}\right)$ followed by a correction step to determine $p\left(\mathbf{X}^{k} \mid \mathbf{Z}^{1: k}\right)$. From $p\left(\mathbf{X}^{k} \mid \mathbf{Z}^{1: k}\right)$ one can determine $p\left(\mathbf{x}_{n}^{k} \mid \mathbf{Z}^{1: k}\right)$ for any particular agent $n$ through marginalization. Note however, that the measurements introduce a posteriori correlation between the states, so that in general $p\left(\mathbf{X}^{k} \mid \mathbf{Z}^{1: k}\right) \neq$ $\prod_{n} p\left(\mathbf{x}_{n}^{k} \mid \mathbf{Z}^{1: k}\right)$.

Comments: In terms of a practical implementation, we observe the following:

- In general, a centralized particle filter [4] can be used to approximate $p\left(\mathbf{X}^{k} \mid \mathbf{Z}^{1: k}\right)$ numerically. The complexity of this particle filter is expected to be high when $N$ is large and when agents perform measurements with respect to other agents. When agents only measure with respect to anchors, $p\left(\mathbf{X}^{k} \mid \mathbf{Z}^{1: k}\right)=\prod_{n} p\left(\mathbf{x}_{n}^{k} \mid \mathbf{Z}^{1: k}\right)$ so that we can run $N$ low-dimensional particle filters, leading to a fully decentralized solution. As an alternative to the particle filter, a linearization of (1) and (2) allows the usage of an extended Kalman filter.

- When the functions $\mathbf{f}_{n}(\cdot)$ and $\mathbf{h}_{n, k}(\cdot)$ are linear and the process and measurement noise are Gaussian, a centralized high-dimensional Kalman filter will provide the optimal solution. When agents only measure with respect to anchors, this solution reverts to one Kalman filter per agent.

- Distributed solutions for the case when agents cooperate have been proposed in [5], [6]. These methods cannot fully capture the a posteriori correlation and are necessarily suboptimal. Distributed Kalman filters were proposed in [7].

\section{B. Bound: Cramér-Rao Bound}

The Cramér-Rao bound (CRB) provides a lower bound on the variance of an unbiased estimator. We will assume $\mathbf{x}_{n}^{k} \in \mathbb{R}^{r}$ and introduce the Fisher information matrix (FIM) of $\mathbf{X}^{0: k} \in \mathbb{R}^{1 \times r N(k+1)}$ as

$$
\mathbf{J}^{0: k}=\mathbb{E}\left\{-\Delta_{\mathbf{X}^{0: k}}^{\mathbf{X}^{0: k}} \log \left(p\left(\mathbf{X}^{0: k}, \mathbf{Z}^{1: k}\right)\right)\right\},
$$

which is an $r N(k+1) \times r N(k+1)$ matrix that has the following property [8], [9]

$$
\mathbb{E}\left\{\left(\hat{\mathbf{X}}^{0: k}-\mathbf{X}^{0: k}\right)\left(\hat{\mathbf{X}}^{0: k}-\mathbf{X}^{0: k}\right)^{\mathrm{T}}\right\} \succeq\left(\mathbf{J}^{0: k}\right)^{-1}
$$

and consequently the CRB is given by

$$
\mathbb{E}\left\{\left\|\hat{\mathbf{X}}^{0: k}-\mathbf{X}^{0: k}\right\|^{2}\right\} \geq \operatorname{tr}\left\{\left(\mathbf{J}^{0: k}\right)^{-1}\right\} .
$$

To determine the lower bound on the localization error of all agents at time $k$, we need to compute the lower right $r N \times$ $r N$ sub-matrix of $\left(\mathbf{J}^{0: k}\right)^{-1}$, denoted by $\left(\mathbf{J}_{\mathrm{E}}^{k}\right)^{-1}$. It is termed the equivalent Fisher information matrix (EFIM) for time $k$. Using Schur's complement, it was shown in [8] that $\mathbf{J}_{\mathrm{E}}^{k}$ can be computed recursively as

$$
\mathbf{J}_{\mathrm{E}}^{k}=\mathbf{C}-\mathbf{B}^{\mathrm{T}}\left(\mathbf{J}_{\mathrm{E}}^{k-1}+\mathbf{A}\right)^{-1} \mathbf{B}
$$


in which

$$
\begin{aligned}
\mathbf{A} & =\mathbb{E}\left\{-\Delta_{\mathbf{X}^{k-1}}^{\mathbf{X}^{k-1}} \log \left(p\left(\mathbf{X}^{k} \mid \mathbf{X}^{k-1}\right)\right)\right\} \\
\mathbf{B} & =\mathbb{E}\left\{-\Delta_{\mathbf{X}^{k-1}}^{\mathbf{X}^{k}} \log \left(p\left(\mathbf{X}^{k} \mid \mathbf{X}^{k-1}\right)\right)\right\} \\
\mathbf{C} & =\mathbb{E}\left\{-\Delta_{\mathbf{X}^{k}}^{\mathbf{X}^{k}} \log \left(p\left(\mathbf{X}^{k} \mid \mathbf{X}^{k-1}\right)\right)\right\} \\
& +\mathbb{E}\left\{-\Delta_{\mathbf{X}^{k}}^{\mathbf{X}^{k}} \log \left(p\left(\mathbf{Z}^{k} \mid \mathbf{X}^{k}\right)\right)\right\}
\end{aligned}
$$

From $\mathbf{J}_{\mathrm{E}}^{k}$, we readily find $\mathbb{E}\left\{\left\|\hat{\mathbf{X}}^{k}-\mathbf{X}^{k}\right\|^{2}\right\} \geq \operatorname{tr}\left\{\left(\mathbf{J}_{\mathrm{E}}^{k}\right)^{-1}\right\}$. Under certain conditions (including $\mathbf{A}, \mathbf{B}$, and $\mathbf{C}$ being constant over time $k$ ), a steady state EFIM $\mathbf{J}_{\mathrm{E}}^{\infty}$ exists, determined by

$$
\mathbf{J}_{\mathrm{E}}^{\infty}=\mathbf{C}-\mathbf{B}^{\mathrm{T}}\left(\mathbf{J}_{\mathrm{E}}^{\infty}+\mathbf{A}\right)^{-1} \mathbf{B} .
$$

Comments: In terms of computing the CRB, we note that $\mathbf{J}_{\mathrm{E}}^{k}$ is still an $r N \times r N$ matrix, which may be hard to compute and invert for large $N$. As in Section III-A, simplifications are possible under some conditions:

- When the functions $\mathbf{f}_{n}(\cdot)$ and $\mathbf{h}_{n, k}(\cdot)$ are linear and the process and measurement noise are Gaussian, the matrices $\mathbf{A}, \mathbf{B}, \mathbf{C}$ have easy expressions similar to those found in the Kalman filter. In addition, the CRB will be tight.

- When agents do not cooperate, $\mathbf{J}_{\mathrm{E}}^{k}$ will be a block diagonal matrix, comprising $N$ blocks of size $r \times r$, so that we can easily track the CRB for each agent individually.

\section{Impact of Timescale SEPARATion}

We are now ready to quantify the impact of timescale separation. For each of the considered impacts, we will work with a simplified scenario that still captures the salient properties of the impact. For ease of exposition, whenever possible, we will drop the subscript referring to the specific agent, i.e., $\mathbf{x}^{k}$, $\mathbf{z}^{k}$.

\section{A. Impact 1: Overly Optimistic CRB}

We consider a non-cooperative scenario and focus on a single agent (so that we can drop the agent index) and use the models described in Section II-B. The agent determines its position in two possible ways:

1) Without separation of timescales: in each period $T_{s}$, the agent performs a measurement with respect to one anchor and updates its posterior. In that case $\log \left(p\left(\mathbf{x}^{k} \mid \mathbf{x}^{k-1}\right)\right) \propto-\left\|\mathbf{x}^{k}-\mathbf{x}^{k-1}-\mathbf{v} T_{s}\right\|^{2} /\left(2 \sigma_{\text {mob }}^{2} T_{s}\right)$ and $\log \left(p\left(\mathbf{z}^{k} \mid \mathbf{x}^{k}\right)\right) \propto-\left\|\mathbf{x}^{k}-\mathbf{x}_{m}\right\|^{2} /\left(2 \sigma_{\text {meas }}^{2}\right)$ in which $\mathbf{x}_{m}$ is the anchor with which the agent ranges at time slot $k$. We then readily find that $\mathbf{A}=\mathbf{I} /\left(\sigma_{\mathrm{mob}}^{2} T_{s}\right)$, $\mathbf{B}=-\mathbf{I} /\left(\sigma_{\text {mob }}^{2} T_{s}\right)$, and $\mathbf{C}=\mathbf{I} /\left(\sigma_{\text {mob }}^{2} T_{s}\right)+\mathbf{I} / \sigma_{\text {meas }}^{2}$.

2) With separation of timescales: the agent collects $K>1$ measurements at the end of each (longer) period of duration $K T_{s}$ and performs a posterior update every $K T_{s}$. In that case $\log \left(p\left(\mathbf{x}^{k} \mid \mathbf{x}^{k-1}\right)\right) \propto-\| \mathbf{x}^{k}-$ $\mathbf{x}^{k-1}-\mathbf{v} K T_{s} \|^{2} /\left(2 \sigma_{\text {mob }}^{2} K T_{s}\right)$ and $\log \left(p\left(\mathbf{z}^{k} \mid \mathbf{x}^{k}\right)\right) \propto$ $-\sum_{m \in \mathcal{M}}\left\|\mathbf{x}^{k}-\mathbf{x}_{m}\right\|^{2} /\left(2 \sigma_{\text {meas }}^{2}\right)$, where $\mathcal{M}$ denotes the set of $|\mathcal{M}|=K$ anchors with which the agent ranges at time slot $k$. Note that now time slots last

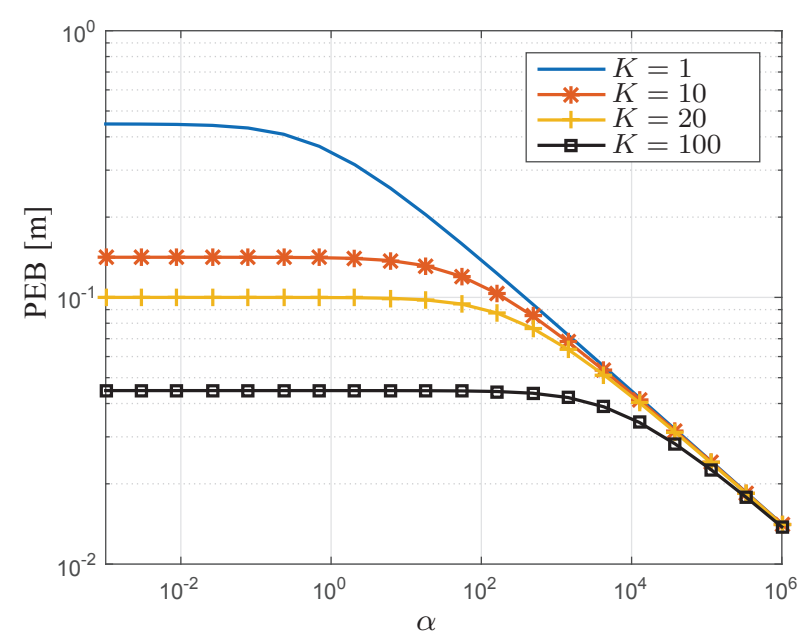

Figure 1. Position error bound (PEB) as a function of $\alpha$ for different values of $K$ and $\sigma_{\text {meas }}^{2}=0.1 \mathrm{~m}^{2}$. The case $K=1$ corresponds to no separation of timescales and leads to the largest PEB.

$K$ times longer than in the first scenario. In this case, $\mathbf{A}=\mathbf{I} /\left(\sigma_{\mathrm{mob}}^{2} K T_{s}\right), \mathbf{B}=-\mathbf{I} /\left(\sigma_{\mathrm{mob}}^{2} K T_{s}\right)$, and $\mathbf{C}=$ $\mathbf{I} /\left(\sigma_{\text {mob }}^{2} K T_{s}\right)+\mathbf{I} K / \sigma_{\text {meas }}^{2}$

When the positioning process starts with a Gaussian symmetric prior, the EFIM will always be a scaled identity matrix of the form $\mathbf{J}_{\mathrm{E}}^{k}=j^{k} \mathbf{I}$. Substitution into (20) and solving for $j^{\infty}$ yields

$$
j_{\mathrm{sts}}^{\infty}=\frac{K}{2 \sigma_{\text {meas }}^{2}}\left(1+\sqrt{1+4 \alpha / K^{2}}\right)
$$

under separation of timescales, where we have introduced $\alpha=$ $\sigma_{\text {meas }}^{2} /\left(\sigma_{\text {mob }}^{2} T_{s}\right)$, and

$$
j_{\mathrm{nsts}}^{\infty}=\frac{1}{2 \sigma_{\text {meas }}^{2}}(1+\sqrt{1+4 \alpha})
$$

when there is no separation of timescales. Note that this latter case corresponds to $K=1$. To obtain a more intuitive result, we introduce the position error bound (PEB), defined as $\mathcal{P}^{\infty}=$ $\sqrt{\operatorname{tr}\left\{\left(\mathbf{J}_{\mathrm{E}}^{\infty}\right)^{-1}\right\}}=\sqrt{2 / j^{\infty}}$ for both cases, expressed in meters. The PEB is shown in Fig. 1 for $\sigma_{\text {meas }}^{2}=0.1 \mathrm{~m}^{2}$ as a function of $\alpha$ for different values of $K$. We see that the separation of timescales leads to a lower PEB, especially for large $K$ and $\alpha$. This means that the design of a positioning system based on a separation of timescales will lead to an overly optimistic performance, when in reality measurements come at a higher rate.

\section{B. Impact 2: Loss of Integrity}

In the above analysis, when $K>1$, it was assumed that all the measurements were available at the end of the period of duration $K T_{s}$. Hence, the CRB did not capture the impact of out of date measurements, i.e., delay between the arrival and processing of the measurement. To address this, we can evaluate the online estimation performance under model mismatch, considering the simplified model from Section II-B: 
1) Without separation of timescales: in each period $T_{s}$, the agent first moves from $\mathrm{x}^{k}$ to $\mathrm{x}^{k+1}$ according to $\mathrm{x}^{k+1}=$ $\mathbf{x}^{k}+\mathbf{v} T_{s}+\mathbf{w}^{k+1}$, with $\mathbf{w}^{k+1} \sim \mathcal{N}\left(0, \sigma_{\mathrm{mob}}^{2} T_{s} \mathbf{I}\right)$ and then performs a measurement $\mathbf{z}^{k+1}=\mathbf{x}^{k+1}-\mathbf{x}_{m}+\mathbf{n}^{k+1}$, with $\mathbf{n}^{k+1} \sim \mathcal{N}\left(0, \sigma_{\text {meas }}^{2} \mathbf{I}\right)$ with respect to an arbitrary anchor (in this case anchor $m$ ) and updates its posterior.

2) With separation of timescales: during each epoch of duration $K T_{s}$ (corresponding to $t^{\prime}+T_{s}, t^{\prime}+2 T_{s}, \ldots, t^{\prime}+$ $K T_{s}$ for some $t^{\prime}$ ), the agent collects the same measurements, say $\mathbf{z}^{k+1}, \ldots, \mathbf{z}^{k+K}$, with $\mathbf{z}^{k+l}=\mathbf{x}^{k}+$ $l \mathbf{v} T_{s}+\sum_{k<l^{\prime} \leq l} \mathbf{w}^{l^{\prime}}-\mathbf{x}_{m}+\mathbf{n}^{k+l}$, in which $\mathbf{w}^{l^{\prime}} \sim$ $\mathcal{N}\left(0, \sigma_{\text {mob }}^{2} T_{s} \mathbf{I}\right)$ and $\mathbf{n}^{k+l} \sim \mathcal{N}\left(0, \sigma_{\text {meas }}^{2} \mathbf{I}\right)$. However, it only processes them at the end of the epoch. Moreover, it treats the measurements as having been acquired at time $t^{\prime}+K T_{s}$, i.e., it assumes $\mathbf{z}^{k+K}=\mathbf{x}^{k+K}-\mathbf{x}_{m}+$ $\mathbf{n}^{k+K}=\mathbf{x}^{k}+K \mathbf{v} T_{s}+\tilde{\mathbf{w}}^{k+K}-\mathbf{x}_{m}+\mathbf{n}^{k+K}$ in which $\tilde{\mathbf{w}}^{k+K} \sim \mathcal{N}\left(0, \sigma_{\mathrm{mob}}^{2} T_{s} K \mathbf{I}\right)$. Hence, there is a mismatch between the actual measurement model and the assumed model.

The model mismatch can lead to positioning errors that are inconsistent with the true posterior distribution and thus lead to a loss of integrity. Integrity of a positioning system refers to the capability to provide bounds on the positioning error. We will consider the same model as in the previous section, which allows us to use a Kalman filter to compute the posterior distribution at each time $k T_{s}$, represented by a Gaussian distribution with mean $\boldsymbol{\mu}^{k}$ and covariance matrix $\boldsymbol{\Sigma}^{k}$. An integrity error occurs when the positioning error cannot be reasonably explained by the state error covariance. Hence, integrity errors happen when

$$
\left(\mathbf{x}^{k}-\boldsymbol{\mu}^{k}\right)^{\mathrm{T}}\left(\boldsymbol{\Sigma}^{k}\right)^{-1}\left(\mathbf{x}^{k}-\boldsymbol{\mu}^{k}\right) \geq \gamma,
$$

where $\gamma$ is a threshold value. Assuming the Kalman filter has converged, we know that the posterior covariance will be $\boldsymbol{\Sigma}=$ $\mathbf{I} / j^{\infty}$. Further assuming that the previous position estimate was correct, the posterior mean when employing separation of timescales will be ${ }^{1}$

$$
\boldsymbol{\mu}^{k}=\mathbf{x}^{k}+K \mathbf{v} T_{s}+\underbrace{\mathbf{K}\left[\mathbf{Z}^{k}-\mathbf{H}\left(\mathbf{x}^{k}+K \mathbf{v} T_{s}\right)\right]}_{\text {error }},
$$

where $\mathbf{H}=\mathbf{1}_{K} \otimes \mathbf{I}_{2}$ is the observation matrix, $\mathbf{K}$ is the (constant) Kalman gain matrix, given by

$$
\begin{aligned}
\mathbf{K} & =\sigma_{p}^{2} \mathbf{H}^{T}\left(\mathbf{H} \sigma_{p}^{2} \mathbf{H}^{T}+\mathbf{I}_{2 K} \sigma_{\text {meas }}^{2}\right)^{-1} \\
& =\frac{1}{K+\sigma_{\text {meas }}^{2} / \sigma_{p}^{2}} \mathbf{H}^{T}
\end{aligned}
$$

in which $\sigma_{p}^{2}=\sigma_{\mathrm{mob}}^{2} T_{s} K+1 / j^{\infty}$, and $\mathbf{Z}^{k}$ is the $2 K \times 1$ vector of stacked measurements. The expected localization error will be the expectation w.r.t. the measurement noise of the error $\mathbf{e}=\mathbb{E}\left\{\mathbf{K}\left[\mathbf{Z}^{k}-\mathbf{H}\left(\mathbf{x}^{k}+K \mathbf{v} T_{s}\right)\right]\right\}$. The expectation of $\mathbf{Z}^{k}-$

\footnotetext{
${ }^{1}$ For notational convenience, we have removed the anchor locations. This does not affect the results.
}

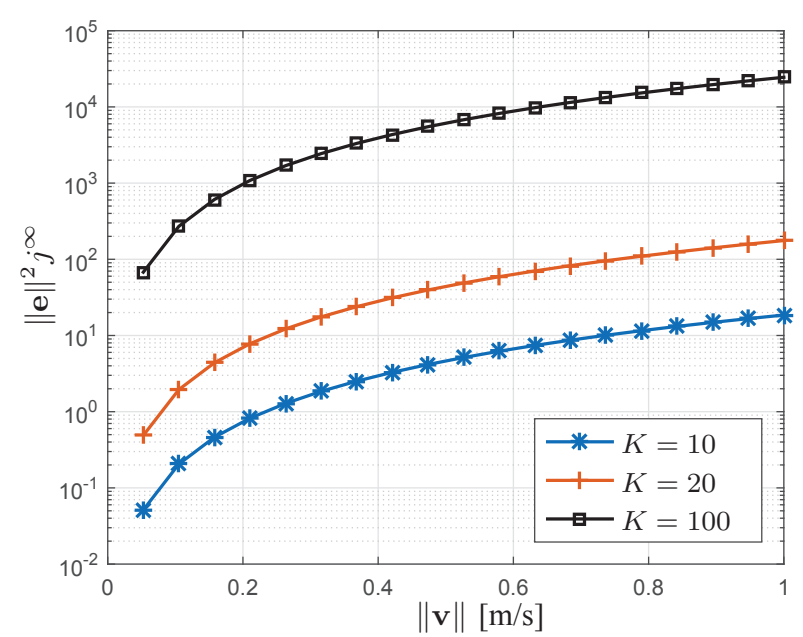

Figure 2. $\|\mathbf{e}\|^{2} j^{\infty}$ as a function of the velocity norm $\|\mathbf{v}\|$ for $T_{s}=0.1 \mathrm{~s}$, $\sigma_{\text {mob }}^{2}=0.1 \mathrm{~m}^{2} / \mathrm{s}, \sigma_{\text {meas }}^{2}=0.1 \mathrm{~m}^{2}$.

$\mathbf{H}\left(\mathbf{x}^{k}+K \mathbf{v} T_{s}\right)$ is a vector obtained by stacking $(K-1) \mathbf{v} T_{s}$, $(K-2) \mathbf{v} T_{s}, \ldots, \mathbf{v} T_{s}, \mathbf{0}$. Hence,

$$
\mathbf{e}=\frac{1}{K+\sigma_{\text {meas }}^{2} / \sigma_{p}^{2}} \frac{K(K-1)}{2} T_{s} \mathbf{v}
$$

Substituting the expected error and the steady-state covariance in (23), we find that integrity errors occur when $\mathbf{e}^{T} j^{\infty} \mathbf{I e}=$ $\|\mathbf{e}\|^{2} j^{\infty} \geq \gamma$, i.e., when

$$
\|\mathbf{v}\| \geq \gamma^{\prime}
$$

where

$$
\gamma^{\prime}=\sqrt{\frac{\gamma}{j^{\infty}}}\left(\frac{\sigma_{\text {meas }}^{2}}{\sigma_{p}^{2}}+K\right) \frac{2}{K(K-1) T_{s}} .
$$

In other words, for velocities below a threshold, we expect no integrity errors to occur. For large velocities, integrity errors will be unavoidable. Note that for $K=1$, integrity errors do not occur. This is illustrated in Fig. 2 showing $\|\mathbf{e}\|^{2} j^{\infty}$ as a function of $\|\mathbf{v}\|$ for different values of $K$. If $\gamma$ is on the order of 10 , we see that for $K=10$, velocities beyond $0.6 \mathrm{~m} / \mathrm{s}$ can be supported, while for $K=20$, velocities less than 0.3 $\mathrm{m} / \mathrm{s}$ are required. Recall that $K T_{s}$ is the time between two measurement updates.

As a numerical example, we consider the case of one anchor and one moving agent. Movement of the agent follows (1) and the measurement model is given by (2) with $\sigma_{\text {mob }}^{2}=0.1 \mathrm{~m}^{2} / \mathrm{s}$, $\sigma_{\text {meas }}^{2}=0.1 \mathrm{~m}^{2}$, and $T_{s}=0.1 \mathrm{~s}$. Measurements arrive every $T_{s}$, but are processed after $K T_{s}$ time, where $K \in\{1,10,20\}$. The agent's initial position is $\mathbf{x}^{0} \sim \mathcal{N}\left(\mathbf{0} \mathrm{m}, 100 \mathbf{I} \mathrm{m}^{2}\right)$. The anchor is randomly positioned with position $\mathbf{x}_{m} \sim \mathcal{N}\left(\mathbf{x}^{k}, 5 \mathbf{I} \mathrm{m}^{2}\right)$. For a given agent velocity $\mathbf{v}$, we let the filter run for 200 timesteps, evaluate (23), and average over 1,000 Monte-Carlo runs. In Fig. 3, distance (23) as a function of the agents velocity is shown. For a given threshold, say $\gamma=10$, velocities up to $0.63 \mathrm{~m} / \mathrm{s}$ can be supported when $K=10$, and for $K=20$ velocities up to $0.23 \mathrm{~m} / \mathrm{s}$ are possible. In the case of $K=1$, 


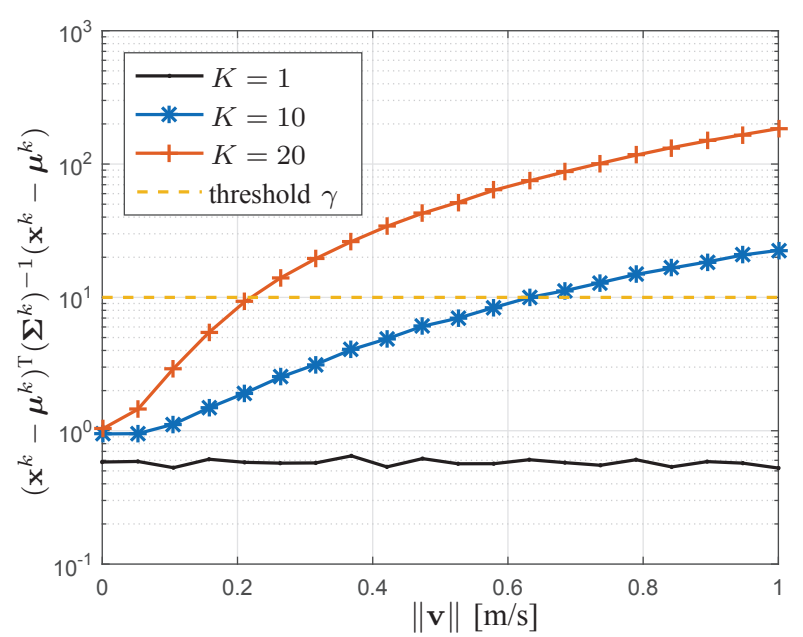

Figure 3. Integrity error as a function of the agents' velocity. The case $K=1$ corresponds to no separation of timescales and does not lead to integrity errors, irrespective of $\mathbf{v}$.

no integrity errors occur and the resulting localization error is only limited by the process and measurement noise.

\section{Impact 3: Overly Confident Beliefs}

The separation of timescales also has an impact on distributed algorithms for cooperative localization. In cooperative localization, the aim is to determine an approximation of $p\left(\mathbf{x}_{n}^{k} \mid \mathbf{Z}_{\leq k}\right)$, called the belief. For the sake of brevity, we focus on two cooperating agents only ${ }^{2}$. Again we have two approaches:

1) Without separation of timescales: The agents have a priori distributions $p\left(\mathbf{x}_{1}^{k}\right)$ and $p\left(\mathbf{x}_{2}^{k}\right)$ and perform two simultaneous measurements $\mathbf{z}_{12}^{k}$ and $\mathbf{z}_{21}^{k}$. Obtaining a measurement generally requires a communication between the two agents. Hence, it is reasonable to assume that $\mathbf{z}_{12}^{k}$ is available to agent 1 while agent 2 has access to $\mathbf{z}_{21}^{k}$. This allows agent 1 to compute $p\left(\mathbf{x}_{1}^{k} \mid \mathbf{z}_{12}^{k}\right)$ and agent 2 to compute $p\left(\mathbf{x}_{2}^{k} \mid \mathbf{z}_{21}^{k}\right)$. Once these computations are completed, both agents move and interact with other agents.

2) With separation of timescales: The agents have a priori distributions $p\left(\mathbf{x}_{1}^{k}\right)$ and $p\left(\mathbf{x}_{2}^{k}\right)$ and perform two simultaneous measurements $\mathbf{z}_{12}^{k}$ and $\mathbf{z}_{21}^{k}$. The agents now perform the SPAWN message passing algorithm [6], where they iteratively update the beliefs $b^{(i)}\left(\mathbf{x}_{1}^{k}\right)$ and $b^{(i)}\left(\mathbf{x}_{2}^{k}\right)$ as follows: we initialize $b^{(0)}\left(\mathbf{x}_{1}^{k}\right)=p\left(\mathbf{x}_{1}^{k}\right)$ and $b^{(0)}\left(\mathbf{x}_{2}^{k}\right)=p\left(\mathbf{x}_{2}^{k}\right)$. Then at iteration $i \geq 1$, agent 1 determines

$$
b^{(i)}\left(\mathbf{x}_{1}^{k}\right) \propto \int p\left(\mathbf{z}_{12}^{k} \mid \mathbf{x}_{1}^{k}, \mathbf{x}_{2}^{k}\right) b^{(i-1)}\left(\mathbf{x}_{2}^{k}\right) \mathrm{d} \mathbf{x}_{2}^{k}
$$

and agent 2 determines

$$
b^{(i)}\left(\mathbf{x}_{2}^{k}\right) \propto \int p\left(\mathbf{z}_{21}^{k} \mid \mathbf{x}_{1}^{k}, \mathbf{x}_{2}^{k}\right) b^{(i-1)}\left(\mathbf{x}_{1}^{k}\right) \mathrm{d} \mathbf{x}_{1}^{k}
$$

\footnotetext{
${ }^{2}$ The extension of additional mobile agents and incorporation of anchors is straightforward.
}

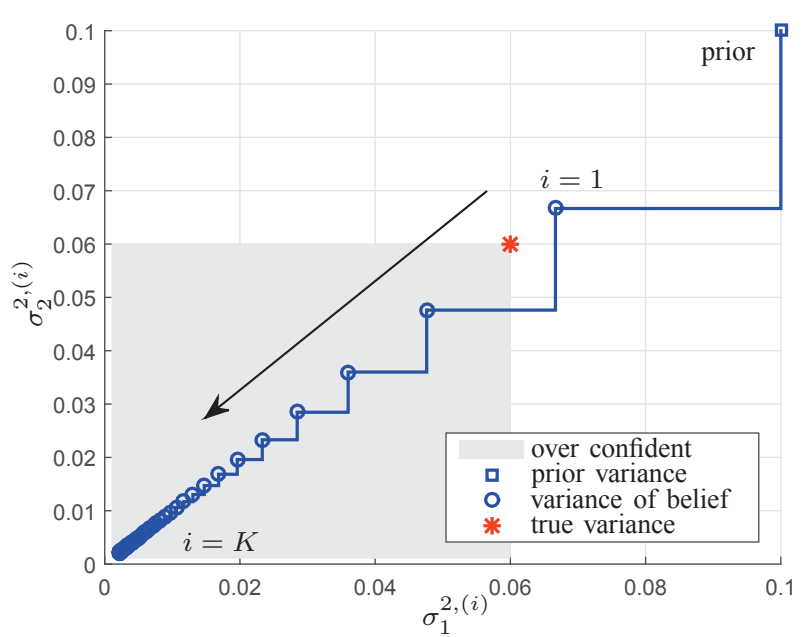

Figure 4. Reduction of uncertainty with increasing number of iterations $i$ in cooperative localization. The resulting variances $\sigma_{1}^{2,(i)}, \sigma_{2}^{2,(i)}$ of agent 1 and 2 are marked by 'o' for every iteration $i$.

We execute $K$ such iterations, after which the update is completed and both agents move and interact with other agents. As before, when $K=1$, we revert to the case without separation of timescales.

In order to analyze this method, we consider again the simplified model from Section II-B and set $p\left(\mathbf{x}_{1}^{k}\right)$ and $p\left(\mathbf{x}_{2}^{k}\right)$ as Gaussians with means $\boldsymbol{\mu}_{1 \text {,prior }}$ and $\boldsymbol{\mu}_{2 \text {,prior }}$ and a common covariance $\sigma_{\text {prior }}^{2} \mathbf{I}$. Then at iteration $i, b^{(i-1)}\left(\mathbf{x}_{1}^{k}\right)$ will be Gaussian with mean $\mathbf{m}_{1}^{(i-1)}$ and covariance matrix $\sigma_{1}^{2,(i-1)} \mathbf{I}$, and similarly for $b^{(i-1)}\left(\mathbf{x}_{2}^{k}\right)$. The updated belief $b^{(i)}\left(\mathbf{x}_{1}^{k}\right)$ is again a Gaussian with mean $\mathbf{m}_{1}^{(i)}$ and covariance $\sigma_{1}^{2,(i)} \mathbf{I}$, given by

$$
\begin{aligned}
\mathbf{m}_{1}^{(i)} & =\sigma_{1}^{2,(i)} \mathbf{I}\left(\frac{\mathbf{m}_{1}^{(i-1)}}{\sigma_{1}^{2,(i-1)}}+\frac{\mathbf{z}_{12}^{k}-\mathbf{m}_{2}^{(i-1)}}{\sigma_{\text {meas }}^{2}+\sigma_{2}^{2,(i-1)}}\right) \\
\sigma_{1}^{2,(i)} & =\frac{\sigma_{1}^{2,(i-1)}\left(\sigma_{2}^{2,(i-1)}+\sigma_{\text {meas }}^{2}\right)}{\sigma_{1}^{2,(i-1)}+\sigma_{2}^{2,(i-1)}+\sigma_{\text {meas }}^{2}}
\end{aligned}
$$

with similar expressions for $b^{(i)}\left(\mathbf{x}_{2}^{k}\right)$.

To understand the behavior of (33), we consider $\boldsymbol{\mu}_{1, \text { prior }}=$ $[0 \mathrm{~m}, 0 \mathrm{~m}]^{\mathrm{T}}, \boldsymbol{\mu}_{2, \text { prior }}=[0 \mathrm{~m}, 5 \mathrm{~m}]^{\mathrm{T}}$ and $\sigma_{\text {prior }}^{2}=0.1 \mathrm{~m}^{2}$, and set $\sigma_{\text {meas }}^{2}=0.1 \mathrm{~m}^{2}$. Applying (33) leads to a reduction of variances $\sigma_{1}^{2,(i)}, \sigma_{2}^{2,(i)}$ with increasing number of iterations $i$. This process is visualized in Fig. 4. We observe that for $K=1$ the variances of the beliefs are overestimated, whereas for $K \gg 1$ they are significantly underestimated. This is a known property of the SPAWN message passing algorithm, when the underlying factor graph contains cycles. Then the beliefs are overly concentrated for large values of $K$.

\section{Conclusions}

In radio-based positioning of mobile nodes, the commonly made assumption of a separation of timescales must be treated 
with care. We have investigated three sources of impact when making this assumption: on the CRB, on out-of-date measurements on tracking, and on message scheduling in cooperative positioning. We found that a positioning system designed on the CRB will be overly optimistic, when in reality measurements arrive at a higher rate. Out-of-date measurements can lead to a loss of integrity in measurement scenarios involving mobility of the agents. Integrity errors occur when the velocity is beyond a certain quantifiable threshold. Finally, we investigated the impact of message scheduling in cooperative positioning, where we found that timescales play an important role in correctly estimating the error covariance.

\section{ACKNOWLEDGMENT}

This work is supported, in part, by the European Research Council under Grant No. 258418 (COOPNET), and by the EU FP7 Marie Curie Initial Training Network MULTIPOS (Multi-technology Positioning Professionals) under Grant No. 316528.

\section{REFERENCES}

[1] G. Garcia, L. Muppirisetty, E. Schiller, and H. Wymeersch, "On the trade-off between accuracy and delay in cooperative UWB localization:
Performance bounds and scaling laws," Wireless Communications, IEEE Transactions on, vol. 13, pp. 4574-4585, Aug. 2014.

[2] D. Simon, Optimal state estimation: Kalman, $H$ infinity, and nonlinear approaches. John Wiley \& Sons, 2006.

[3] S. Van de Velde and H. Steendam, "On the effect of movement on the localization accuracy in practical wireless networks," in IEEE 20th Symposium on Communications and Vehicular Technology in the Benelux (SCVT), Nov. 2013

[4] M. S. Arulampalam, S. Maskell, N. Gordon, and T. Clapp, "A tutorial on particle filters for online nonlinear/non-Gaussian Bayesian tracking," IEEE Transactions on Signal Processing, vol. 50, no. 2, pp. 174-188, 2002

[5] A. Ihler, J. Fisher, R. Moses, and A. Willsky, "Nonparametric belief propagation for self-localization of sensor networks," IEEE Journal on Selected Areas in Communications, vol. 23, pp. 809-819, Apr. 2005.

[6] H. Wymeersch, J. Lien, and M. Z. Win, "Cooperative localization in wireless networks," Proceedings of the IEEE, vol. 97, pp. 427-450, Feb. 2009.

[7] R. Olfati-Saber, "Distributed Kalman filtering for sensor networks," in 46th IEEE Conference on Decision and Control (CDC), pp. 5492-5498, Dec. 2007.

[8] P. Tichavsky, C. Muravchik, and A. Nehorai, "Posterior Cramér-Rao bounds for discrete-time nonlinear filtering," IEEE Transactions on Signal Processing, vol. 46, pp. 1386-1396, May 1998.

[9] S. Kay, Fundamentals of Statistical Signal Processing: Estimation theory. Fundamentals of Statistical Signal Processing, Prentice-Hall PTR, 1993. 